\title{
Multi-layer topology control for long-term wireless sensor networks
}

\author{
Ikjune Yoon ${ }^{1}$, Dong Kun Noh ${ }^{2^{*}}$ and Heonshik Shin ${ }^{1}$
}

\begin{abstract}
Due to the inefficiency of a flat topology, most wireless sensor networks (WSNs) have a cluster or tree structure; but this causes an imbalance of residual energy between nodes, which gets worse over time as nodes become defunct and replacements are inserted. Multiple layers are better then the typical two-layer cluster-based topology, because it can better accommodate nodes with different levels of residual energy. We propose that each node should periodically determine its own layer, as its situation and the network topology changes. We introduce a topology control scheme for long-term WSNs with these features. Simulations show that this scheme can balance node energy levels, and thus extend network lifetime.
\end{abstract}

Keywords: hierarchical topology, network lifetime, wireless sensor network, topology control, energy adaptive routing, long-term WSN

\section{Introduction}

Simple wireless sensor networks (WSNs) usually have a flat topology and transmit data using a flooding scheme, of which there are several variants. However, flooding can cause the broadcast storming problem [1], reducing the efficiency and reliability of the WSN.

Hierarchical topology control (TC) schemes [2-5] are designed to overcome the broadcast storming problem and to support in-network processing, which improves network performance. A drawback of hierarchical TC schemes is that an imbalance in residual energy between aggregation nodes and general nodes can occur over time, since the aggregation nodes can be expected to consume energy faster than the others [6]. It is even possible for some aggregation nodes to cease participation in the network because they lack energy. In previous schemes $[2,4,7]$, this problem has been overcome by getting nearby energy-rich nodes to take over the roles of defunct aggregation nodes; or new aggregation nodes must be deployed to extend the lifetime of the WSN.

In a WSN which uses hierarchical TC and operates for a long period, the imbalance in residual energy between the nodes can become serious as the number

\footnotetext{
* Correspondence: dnoh@ssu.ac.kr

${ }^{2}$ School of Electronic Engineering, Soongsil University, Seoul, Korea

Full list of author information is available at the end of the article
}

of failed and insertions or removals replacement nodes recounts of [8]. Draconian changes to the network topology are also likely to be necessary over time.

We propose new TC scheme designed specially for WSNs which are to be maintained for a long period. It minimizes the variation in residual energy between nodes, and thus extends the network lifetime. This goal is achieved by replacing the usual one- or two-layered topology with multiple layers, which can accommodate a wide range of node energy levels more precisely. This new scheme also gets nodes to change roles dynamically as the energy and traffic context changes. This is necessary because the energy level of each node and the network topology can both change radically in long-term WSNs. In our scheme, each node periodically determines its own layer in response to its energy status, with traffic and topology information. There has been a lot of research on hierarchical topologies for WSNs; but, to the best of our knowledge, this is the first contextaware multi-layer TC scheme for long-term WSNs.

The rest of this article is organized as follows: We explain the outline of the hierarchical TC and its problems in the next section. In Section 3 we describe our layer-based TC scheme for long-term WSNs. We then evaluate the performance of our algorithm in Section 4 and draw conclusions in Section 5. 


\section{Background}

\subsection{Preliminaries}

Hierarchical TC schemes have been shown to produce considerable savings in the total energy consumption of a WSN because of data aggregation [7]. In a hierarchical TC scheme, the nodes are clustered and a head node is assigned to each cluster. A head node is the leader of its group, with the primary responsibility of collection and aggregation of data from its cluster, and transmission of the aggregated data to the sink. Data aggregation greatly reduces energy consumption, because it reduces the total number of messages to be sent to sink, and hence extends network life time. Clustering involves a configuration and maintenance overhead; but in practice it has been demonstrated that clustering improves both energy consumption and performance in large WSNs.

Clustering is not without its problems: a node that cannot act as a cluster head and is not near to a cluster head becomes void, as shown in Figure 1a; and the cluster heads consume much more energy than the other nodes, leading to an imbalance across the network. This effect also occurs in a tree-based hierarchical topology, as nodes near the sink node have to transmit considerably more data than those at the rim of the network [9], as shown in Figure 1b.

\subsection{Related work}

Oyman and Ersoy [8] investigated the choice of multiple sinks. They use a cluster scheme in which the sinks act as cluster-heads, and each node only reports to one cluster-head. Das and Dutta [10] proposed a similar method of sink selection, designed to minimize overall energy consumption.

Buratti et al. [11-13] considered the reachability of multiple sinks in tree-based topologies with fixed node and sink densities.

Kim and Lee [14], and Fan et al. [15] used a spanning tree to control a topology in multi-sink WSNs. Kim and Lee's scheme reconfigures itself automatically when nodes fail, increasing network lifetime.

Fan et al. [15] proposed a three-layer topology for large-scale WSNs in which each layer has its own role. They provide an algorithm that finds all the bottleneck nodes and eliminates them.

Ciciriello et al. [16] introduced a scheme in which the search for a multi-sink topology is mapped to a multicommodity network design problem. This scheme periodically adapts message paths to reduce the number of network links that are used, increasing the efficiency of data transmission.

Intanagonwiwat et al. [17] create a tree which is limited to a single sink at its root. Paths from sensor nodes nearer to the sink are added earlier gradually.

Other authors [18-20] have also utilized a three-layer topology as the basis for control algorithms for static WSNs. Sharma et al. [18] create high-performance clusters and zonal sink nodes in order to increase the lifetime of sensor nodes. Duan et al. [19] used several

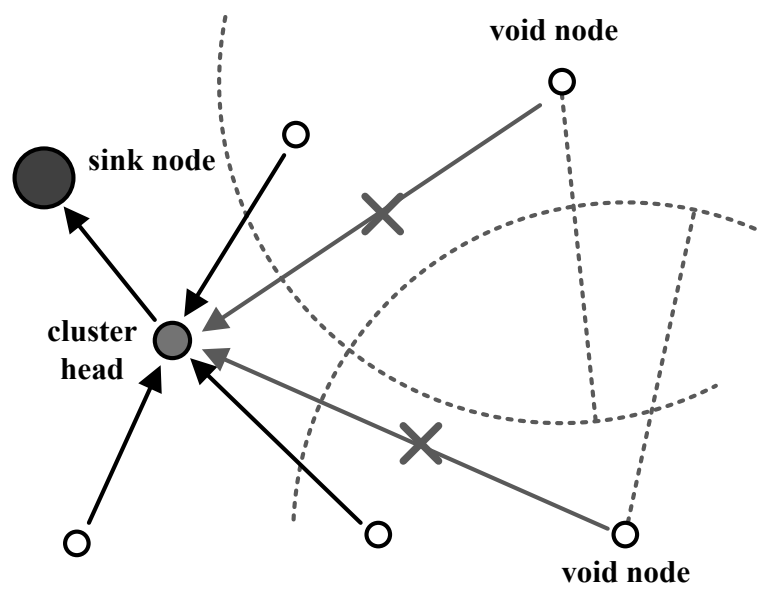

(a) Void problem in a cluster topology

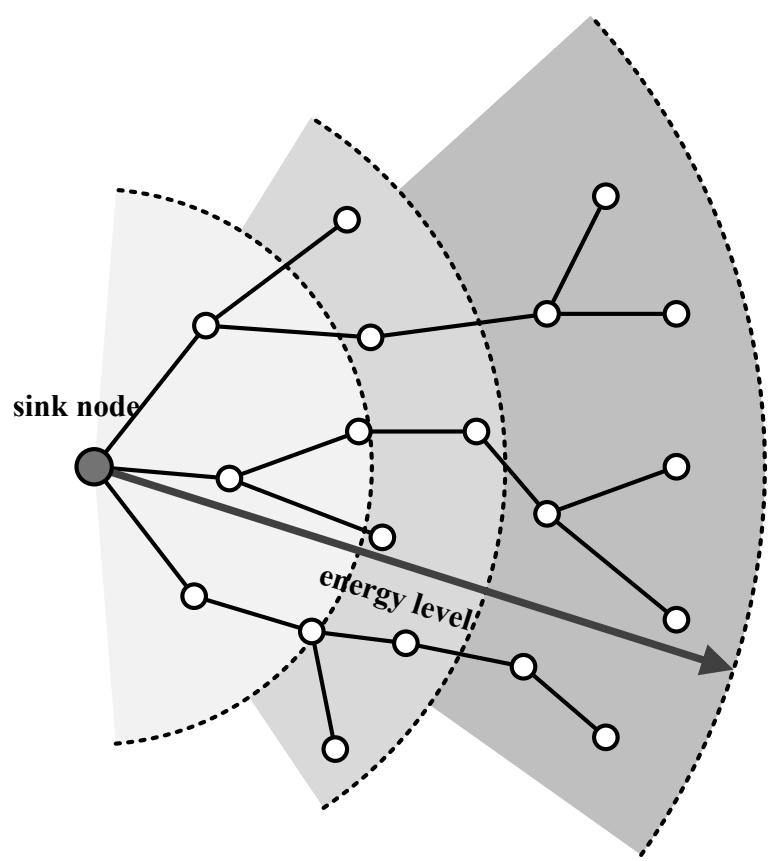

(b) Energy imbalance of tree topology

Figure 1 Problems in cluster and tree topologies. Void problem in a cluster topology and energy imbalance of tree topology. 
fusion nodes and a control node to reduce the energy consumption of mobile nodes. Ming et al. [20] introduced a logical three-tiered TC model which consist of relay nodes, application nodes and sensor nodes. Their cluster-heads organize themselves into a near-uniform distribution across the network.

\section{Layer-based TC}

We will now introduce the architecture of our scheme and explain how the nodes decide which layer they should belong to. The network we will discuss is composed of battery-based sensor nodes, each of which periodically transmits the data it has acquired to a sink node.

\subsection{Proposed scheme}

Each node selects a layer to join from its context, which is primarily its residual energy and the amount of traffic it has to handle, and the nodes in each layer treat the nodes in higher layers as sinks, as they would in a multi-sink network. Figure 2 shows a simple three-layer topology that might be constructed at a particular time by our scheme. If a node can send its data to an upperlayer node directly, it does so; otherwise it sends it to a neighbor on the same layer for relaying. In Figure 2, for example, nodes $n_{3}, n_{5}, n_{7}, n_{8}$ and $n_{10}$ in Layer 2 send data to the upper-layer nodes. Node $n_{5}$, for example, can send data to a higher-layer node directly; but nodes $n_{1}$ and $n_{2}$ have to transmit data through neighboring nodes.
Since our scheme uses same-layer nodes for relaying, fewer void nodes are caused by the absence of cluster heads, as shown in Figure 3. We will explain this in more detail in Section 3.2.

\subsection{The layering algorithm design}

We consider each node $n_{i}$ to have a set $N_{i}$ of neighbor nodes and each node sends data towards the sink node $s$ at the end of every period $P_{G}$. The sink node $s$ broadcasts topology control information (TCI) messages at the start of every setup period $P_{S}$ by using data flooding. This data determines the layer of each node.

Figure 4 shows the stages in our algorithm. First, the sink node $s^{0}$ (in Layer 0) broadcasts a TCI message for the current round, of length $P_{S}$. This message is composed by the sink node, using information gathered from all the nodes in the WSN before the setup period. We assume that $P_{S}$ is even greater than $P_{G}$ because our target is long-term WSN in which layer selection is rarely occurred. Thus the overhead of flooding TCI message is little enough to be negligible.

A node $n_{i}$ can determine its layer $j$ from this information, and can be written $n_{i}^{j}$. If a node $n_{i}^{j}$ receives another TCI message from a node in a layer higher than $j$, it re-determines its layer from the new TCI message; but this layer must not be higher than the layer of the sender of the TCI message. Otherwise, the node would become void, as shown in Figure 5, and the data sent to it from lower-layer nodes would be lost. After it has determined its layer, a node adds that information to

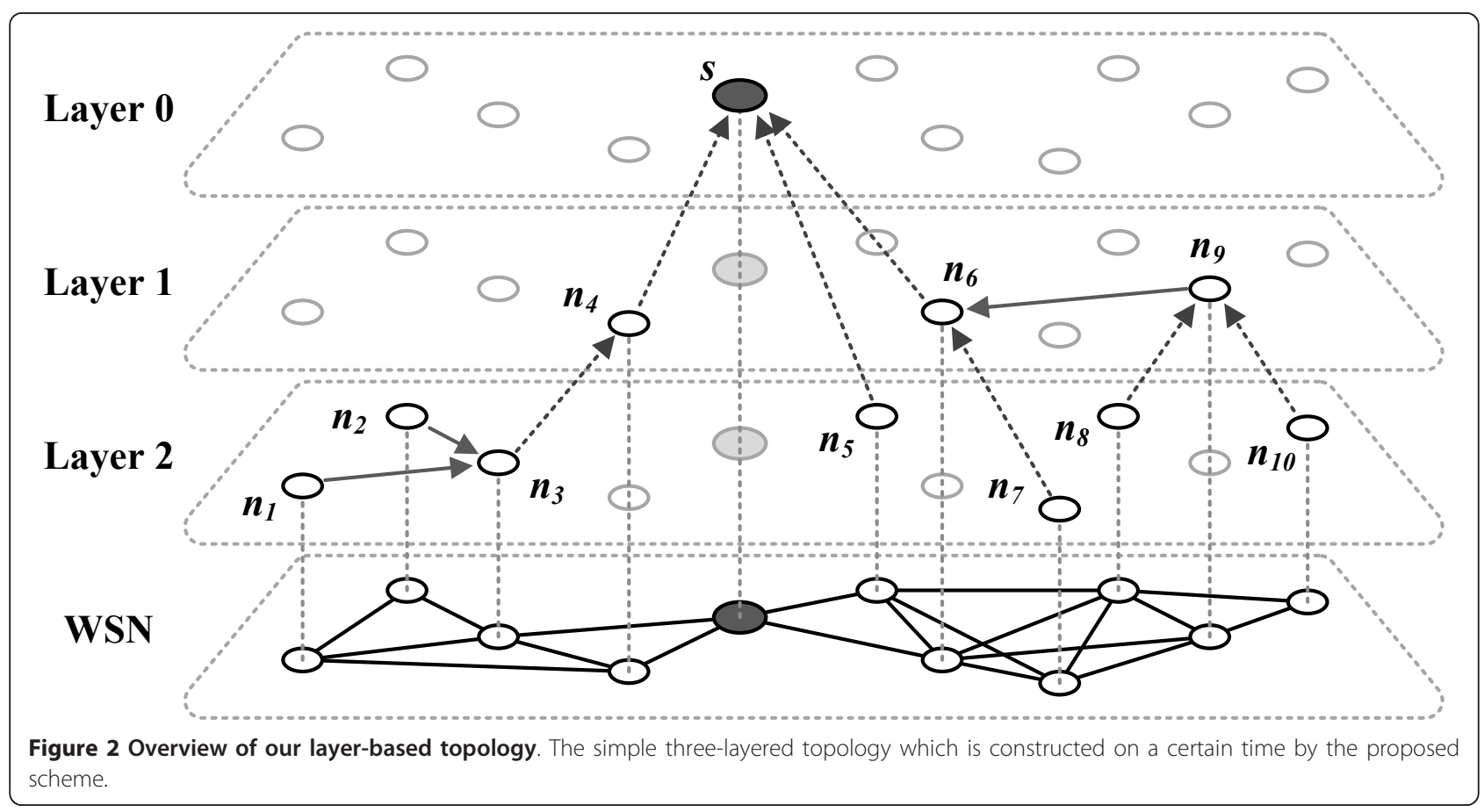




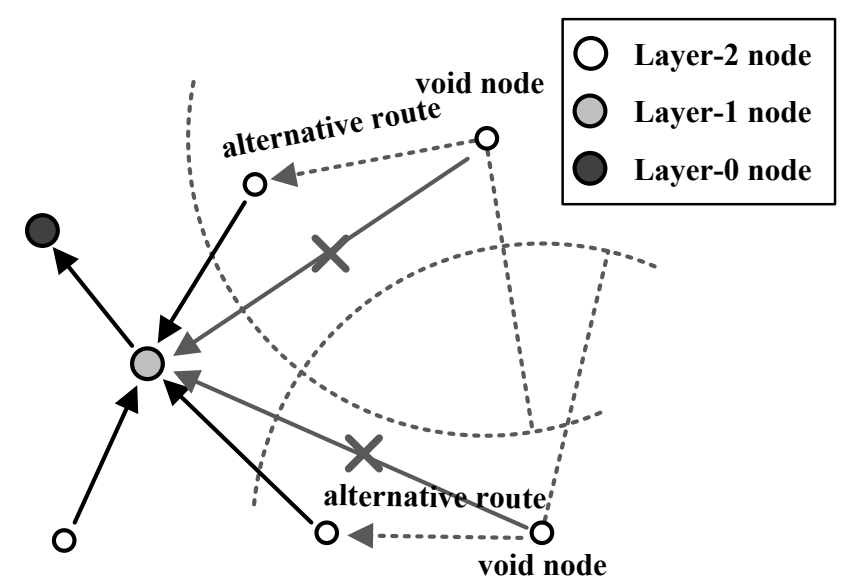

Figure 3 Prevention of void nodes. Our scheme avoids void node problem by using same-layer nodes as a relaying node.

the TCI message it received, and broadcasts the revised message to its neighbors. Repetition of this process makes it possible for all the nodes to determine their own layer. Algorithm 3.2 is a formal statement of this procedure.

\section{Algorithm 1 Layer-based TC algorithm}

Require: Sink node $s$ broadcasts the TCI message.

Require: The layer $j$ of each node is MAX_LAYER

Require: $s$ calculates $\bar{R}$, the average expected lifetime of all nodes.

Ensure: Determining the layer $j$ for $n_{i}^{j}$

1: if $n_{i}^{j}$ receives the TCI message from $n_{k}^{a}$ then

2: if $j>a$ then

3: $l \leftarrow j$

4: $j \leftarrow a$

5: while $j<l$ do

6: calculating the estimated lifetime $R_{i}^{j}$ of $n_{i}^{j}$ \{Equation (6)\}

7: $\quad$ if $R_{i}^{j}>\bar{R}$ then
8: broadcasting the TCI message $\{j$ is selected as the new layer for $n_{i}^{j}$ \}

$$
\begin{aligned}
& \text { 9: } \quad \text { return } \\
& \text { 10: } \quad \text { else } \\
& \text { 11: } \quad j \leftarrow j+1 \\
& \text { 12: } \quad \text { end if } \\
& \text { 13: } \quad \text { end while } \\
& \text { 14: } \text { end if } \\
& \text { 15: } \text { end if }
\end{aligned}
$$

\subsection{Layer determination}

Each node determines its layer by comparing its expected lifetime with the average lifetime of all the nodes. We will now explain this layer-determination algorithm in detail.

\subsubsection{TCl message}

All the nodes in the WSN send the information described in Table 1 to the sink node $s$, so that it can prepare the TCI message. From this information the sink node calculates $C^{j}$, the number of nodes currently in each layer, the average lifetime of all nodes, $R$, and

\section{sending $\mathrm{TCI}$ message}

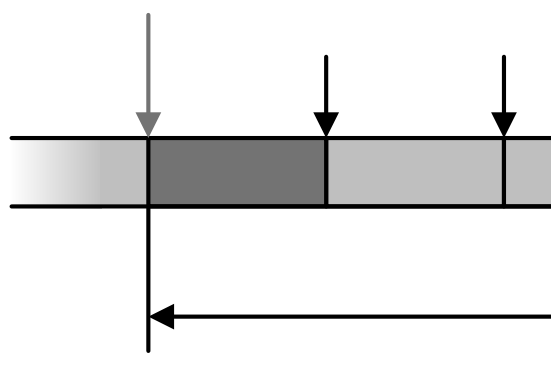

Figure 4 Stages in the TC process

\section{gathering sensory data \\ sending $\mathrm{TCI}$ message}




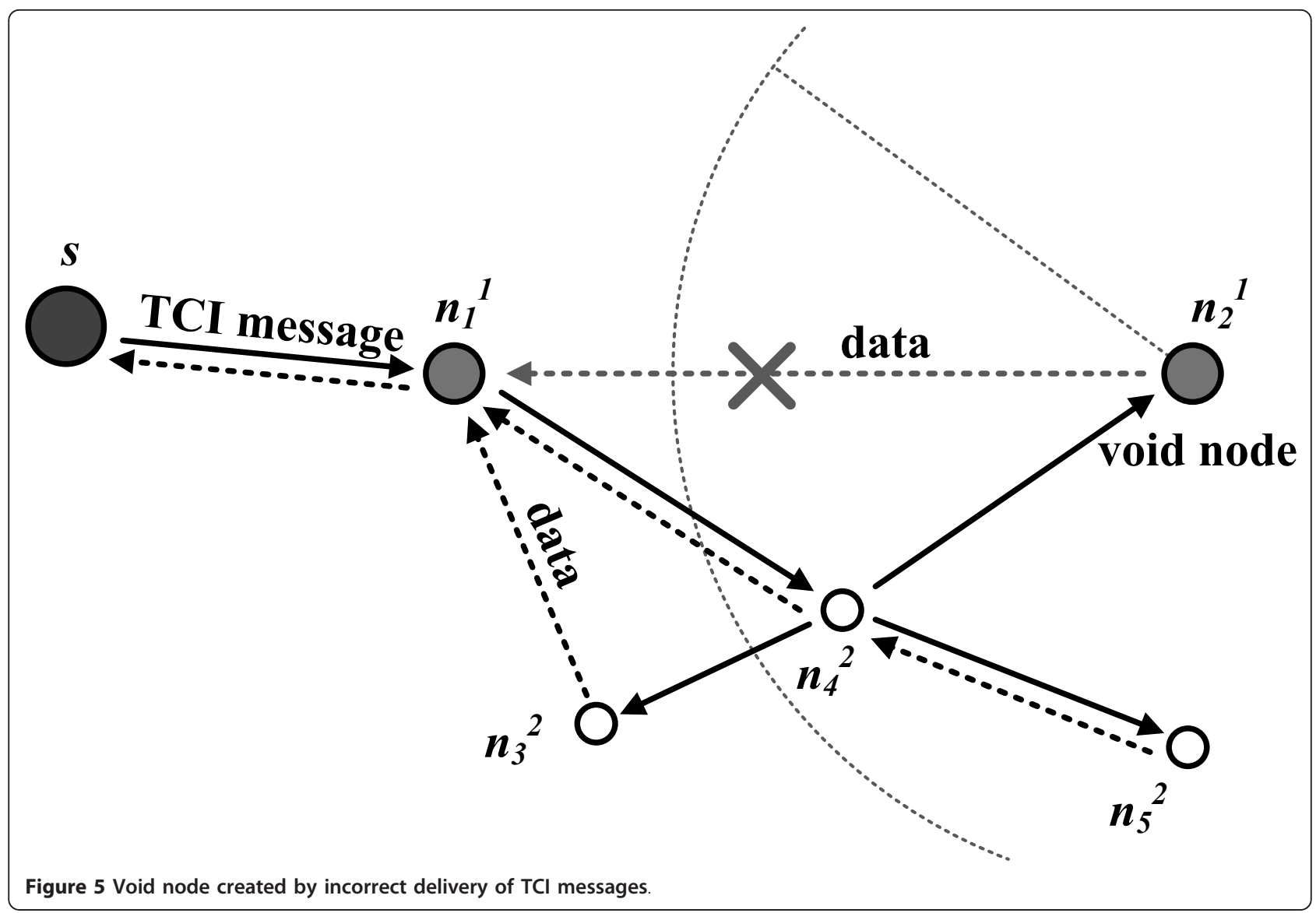

the expected amount of data that each layer should relay, $\bar{L}_{\text {relay }}^{j}$. This information is put into the TCI message; Table 2 describes all the information contained in a TCI message.

\subsubsection{How a node selects its layer}

A node receiving a TCI message determines its layer by comparing $\bar{R}$ with its expected lifetime. This is calculated from the following energy model [21]:

$$
e_{\text {consume }}=e_{\text {trans }}+e_{\text {receive }}+e_{\text {elec }}
$$

where $e_{\text {trans }}$ and $e_{\text {receive }}$ respectively are the amount of energy consumed when the node sends and receives packets, and $e_{\text {elec }}$ is the energy consumed by the electronics. If packets are transmitted for $P$ seconds and the transmission range is tr, the amount of energy

Table 1 Node information required in preparing a $\mathrm{TCl}$ message

\begin{tabular}{ll}
\hline Notation & Descriptions \\
\hline$j$ & Number of layers in which $n_{i}^{j}$ is present \\
$R_{j}^{j}$ & Expected lifetime of $n_{i}^{j}$ (in rounds) \\
$L_{\text {relay }}^{j}$ & $\begin{array}{l}\text { Amount of data that node } n_{i}^{j} \text { should relay on layer } j \text { during } \\
\text { one round }\end{array}$ \\
\hline
\end{tabular}

consumed is

$$
\int_{P} e_{\text {consume }}=L_{\text {trans }} \beta t r^{\alpha}+\int_{P}\left(e_{\text {receive }}+e_{\text {elec }}\right) \text {, }
$$

where $L_{\text {trans }}$ is the number of bits in packets transmitted for $P$ seconds, $\alpha$ is the path loss $(2 \leq \alpha \leq 5)$, and $\beta$ is the energy used by the power amplifier for transmitting 1 bit over a distance of $1 \mathrm{~m}$. Thus we can calculate the energy consumed in one round by a node $n_{i}$ belonging to layer $j$, as follows:

$$
e_{\text {round }_{i}^{j}}^{j}\left\lfloor\frac{P}{P_{G}}\right\rfloor L_{\text {trans }}^{j} \beta t r_{i}^{\alpha}+\int_{P_{S}}\left(e_{\text {receive }}+e_{\text {elec }}\right) .
$$

Table 2 Information in a $\mathrm{TCl}$ message

\begin{tabular}{ll}
\hline Notation & Descriptions \\
\hline$j$ & Number of the layer in which the sender resides \\
$C$ & Total number of nodes in each layer \\
$\bar{R}$ & $\begin{array}{l}\text { Expected lifetime of the entire WSN (in rounds) } \\
\bar{L}_{\text {relay }}\end{array}$ \\
$M$ & $\begin{array}{l}\text { Amount of data that each layer should relay during one } \\
\text { round }\end{array}$ \\
\hline
\end{tabular}


$L_{\text {trans }}^{j}$ is the size of a packet, which is the sum of the packet header size $L_{\text {head }}$, the amount of data $c_{i}^{j}$ received from a lower layer, and the amount of data $L_{\text {relay }}^{j}$ to be relayed from nodes on the same layer, as follows:

$$
L_{\text {trans }}^{j}=L_{\text {head }}+\left(L_{\text {data }} c_{i}^{j}\right)+L_{\text {relay' }}^{j}
$$

where $c_{i}^{j}$ is the number of lower-layer nodes that send data to $n_{i}^{j}$. However, we cannot measure the value of $c_{i}^{j}$ accurately because it can change with the topology. Therefore we estimate $c_{i}^{j}$ from $h_{i}^{j}$, the number of nodes in each layer that sent data to $n_{i}^{j}$ during a previous round, and $N^{j}$, the total number of nodes in each layer, as follows:

$$
c_{i}^{j}=\frac{\sum_{k=j+1, h_{i}^{k}=-1}^{M} N^{k}}{\sum_{k=0}^{j} N^{j}+1}+\sum_{k=j+1, h_{i}^{k} \neq-1}^{M} h_{i}^{k}, \quad \text { initial } h_{i}^{k}=-1
$$

If use replace $L_{\text {relay }}^{j}$ in Equation 4 with $\bar{L}_{\text {relay }}^{j}$, and use an average path loss calculated during a previous round as $\alpha$ because we can not get an accurate value, then we use the modified equation to determine the energy consumed by node $n_{i}^{j}$ in layer $j$ during the current round. The lifetime of $n_{i}^{j}$ can be calculated from $e_{\text {round }_{i}^{j}}$ as follows:

$$
R_{i}^{j}=\left\lfloor\frac{e_{\text {remain } i^{j}}}{e_{\text {round }_{i}^{j}}}\right\rfloor
$$

This computation is repeated for decreasing values of $j$ : the first value of $j$ which satisfies $R_{j}^{j}>\bar{R}$ determines the layer of the node. If this inequality is never satisfied, the node enters the lowest layer $M$. Getting nodes to choose their layers dynamically in this way moves nodes with a lot of energy into higher layers, where they will work harder, while nodes that are nearly exhausted go to lower layers, where they can conserve energy.

\section{Simulation}

We wrote a simulation in $\mathrm{C}++$ to evaluate the performance of the proposed scheme.

\subsection{Simulation environment}

From the simulation results, we computed the average lifetime of sensor nodes, and its standard deviation, the average number of data packets transmitted, and the number of dead nodes. We considered a flat network topology, and two, three, and four layers. We simulated a network of 1,000 nodes at a density of 0.02 to 0.1 nodes $/ \mathrm{m}^{2}$, and each test set was run for 6,000 rounds and repeated ten times; then the results use averaged. At the beginning of each round, all nodes were classified into layers (except for the flat topology) using Algorithm 3.2. For example, Figure 6 shows the distribution of nodes in each of three layers. The nodes transmitted data to a sink node using the AODV algorithm [22] at an interval of $P_{G}$. Each sink node broadcasts a TCI message (similar to a Routing REQuest message in AODV) and all nodes send their data along the reverse of the path that TCI message pass through (similar to a Routing REPly message in AODV). Then the nodes in a higher layer aggregate the data received from the lowerlayer nodes with their own data, and transmit all the data to the next node. Figure 7 depicts an example of our simulation process. Each node is powered by a battery of finite life, and dead nodes are immediately replaced with new nodes in the same position. Table 3 contains the important parameters used in our simulation.

\subsection{Simulation results}

Figures 8 and 9 respectively show the number of packets in the network and the average lifetime of the nodes, measured from our simulation. Figure 9 shows that the layered topologies increased the average lifetime of a node by 2.1 to $22 \%$, compared to the flat topology. In general more layers give a higher performance. Figure 8 shows that the number of packets transmitted decreased by between 30 and $50 \%$ as the density of the nodes increased. As node density decreases, the opportunity to receive a TCI message direct from a higher layer decreases. Thus there are fewer nodes in the higher layers, and there is less data aggregation. Conversely, as the density of nodes increases, the fewer packets are transmitted, and the lifetime of the WSN increases, as shown in Figure 8. Our simulation used a simple data

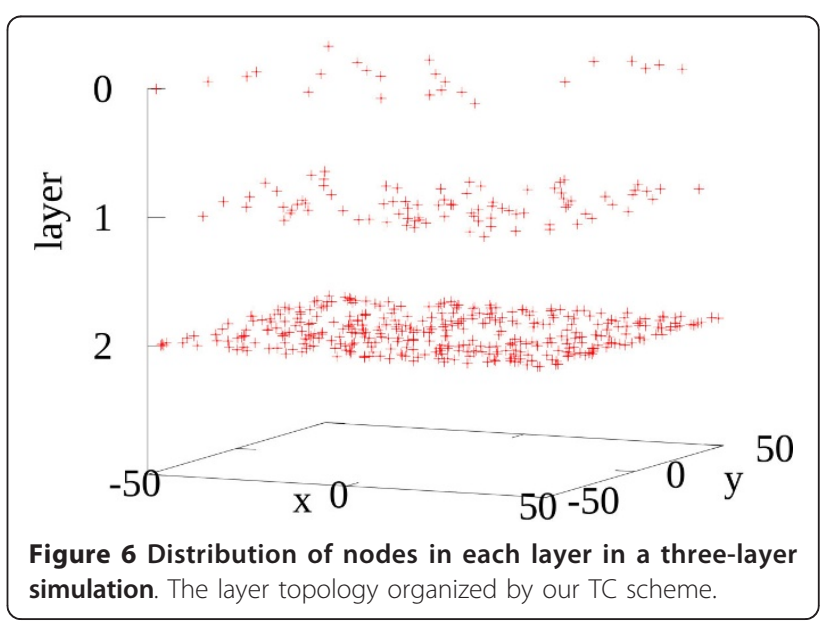




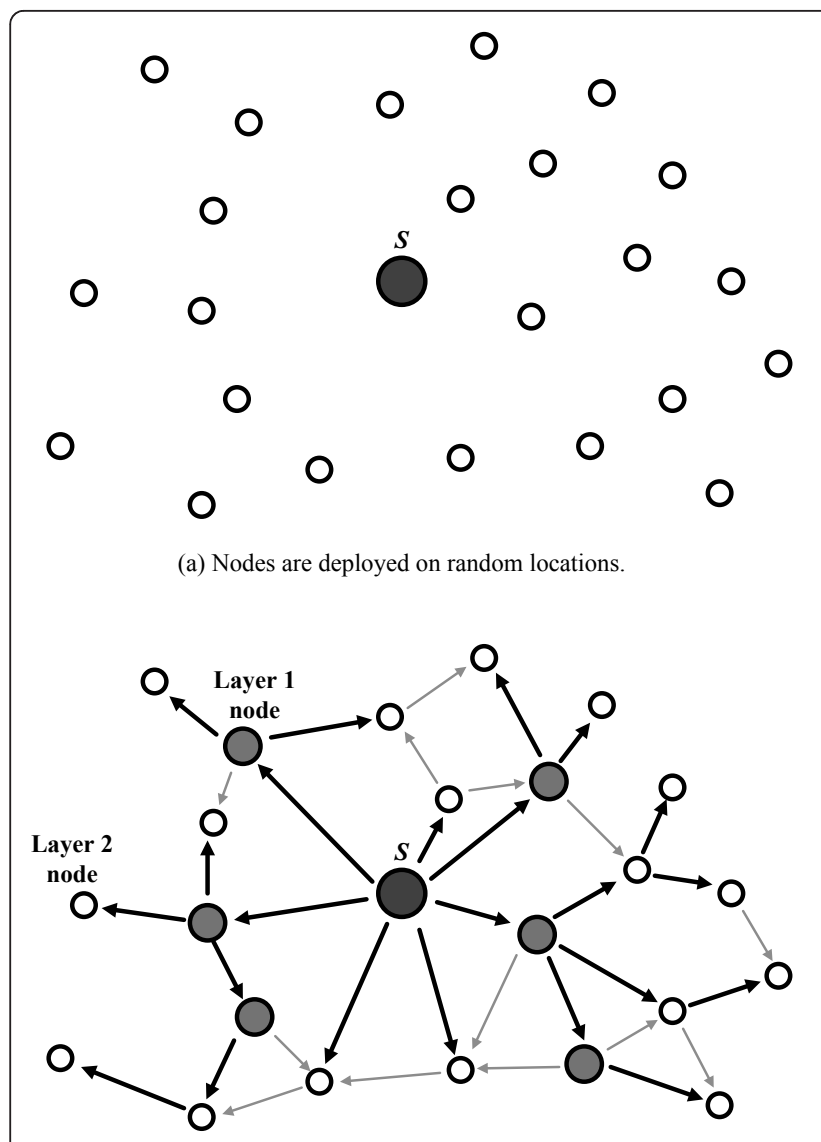

(c) Nodes select their own layers and reverse paths.

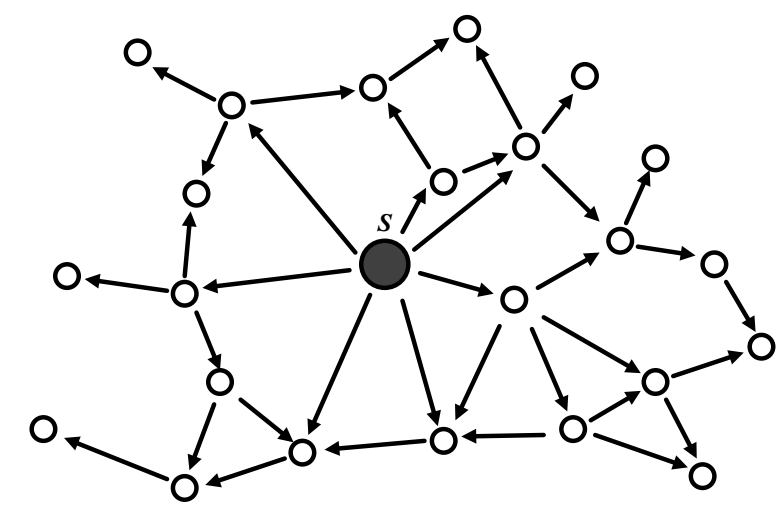

(b) Sink node $s$ floods TCI messages

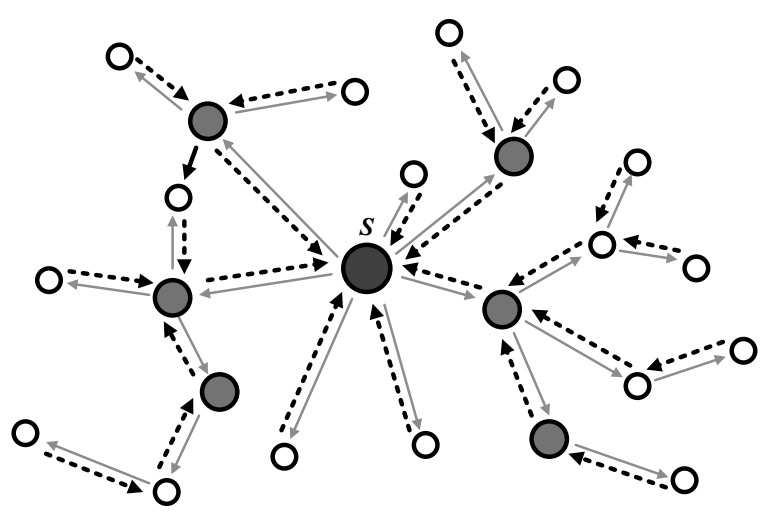

(d) Nodes send data to sink node $s$ along the reverse paths

Figure 7 An example of our simulation process.

aggregation strategy, in which a node which receives data from a lower layer removes the headers and forwards the incoming data with its own data. This strategy only eliminates the packet headers received from lowerlayer nodes, and thus the average lifetime of the WSN did not increase very greatly. A more efficient aggregation scheme would have much more effect on the amount of data to be sent to upper-layer nodes and we could expect the lifetime of the WSN to improve significantly.

Figure 10 shows the standard deviation of the lifetime of nodes. This is quite high because the nodes closer to the sink node have a shorter lifetimes since they have to relay more data. Depending on the density of the nodes, the standard deviation is reduced by 9 to $36 \%$ by our scheme, compared to the flat topology.

Figure 11 shows the cumulative number of dead nodes. Using the flat topology, many nodes died early because of the high standard deviation of lifetime, whereas nodes separated into layers survived longer. This shows how our scheme can prolong the lifetime of a WSN.
Our scheme makes adjusts the number of layers adaptively, in response to the changing situations of the nodes, up to a specified maximum. Our experiments suggest that performance is usually improved by increasing the maximum number of layers, up to a certain number, after which there is no further improvement. For example, in Figure 9, when the density is 0.1, the average lifetime of a node is not increased by going

Table 3 Important simulation parameters

\begin{tabular}{ll}
\hline Parameter & Value \\
\hline Number of nodes & 1,000 \\
Node density & $0.02-0.1$ \\
Node placement & Random \\
Amount of data of a packet & 100 bytes \\
Size of header of a packet & 40 bytes \\
Transmission range & $10-20 \mathrm{~m}$ \\
Maximum number of layers & $1-4$ \\
$\alpha$ and $\beta$ in Equation (2) & 3 and $100 \mathrm{pJ} / \mathrm{bit} / \mathrm{m}^{2}$ \\
$e_{\text {elec }}$ and $e_{\text {receive in Equation }(2)}$ & 0.003 and $0.066 \mathrm{~J}$ \\
$P_{S}$ and $P_{G}$ in Equation (3) & 1,000 and $100 \mathrm{~s}$ \\
\hline
\end{tabular}




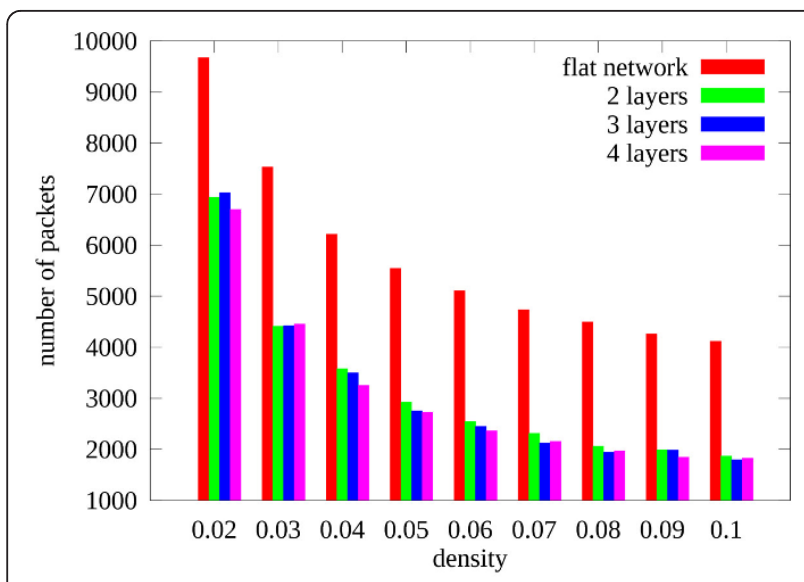

Figure 8 Number of packets transmitted. The number of gathered packets in our simulation according to the density of nodes.

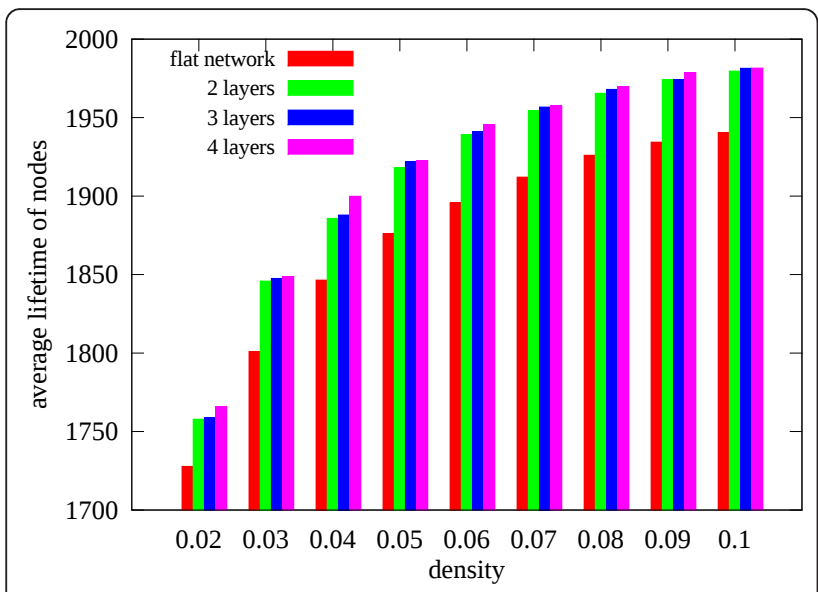

Figure 9 Average lifetime of nodes. The average lifetime of the nodes measured in our simulation according to the density of nodes.

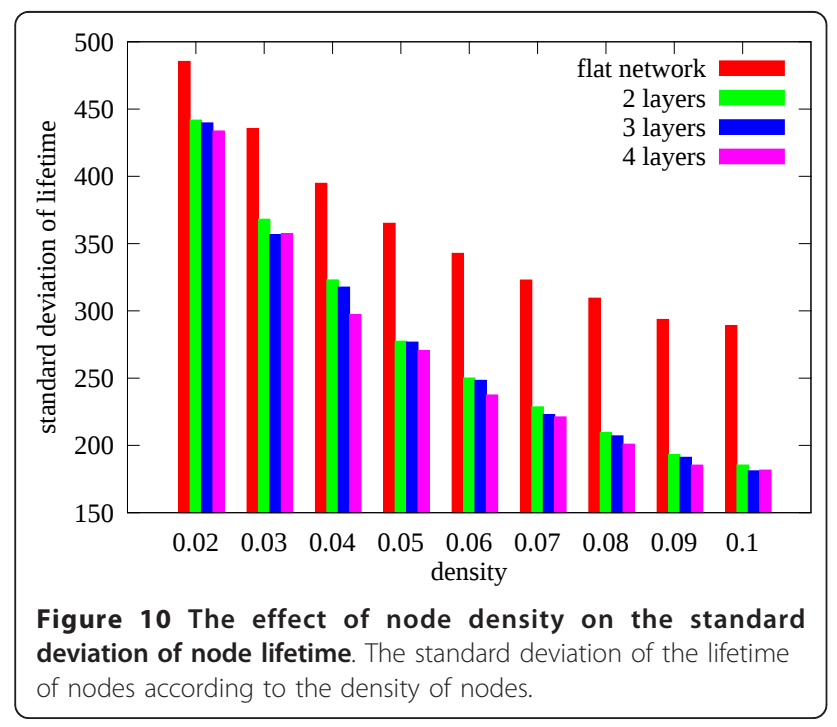

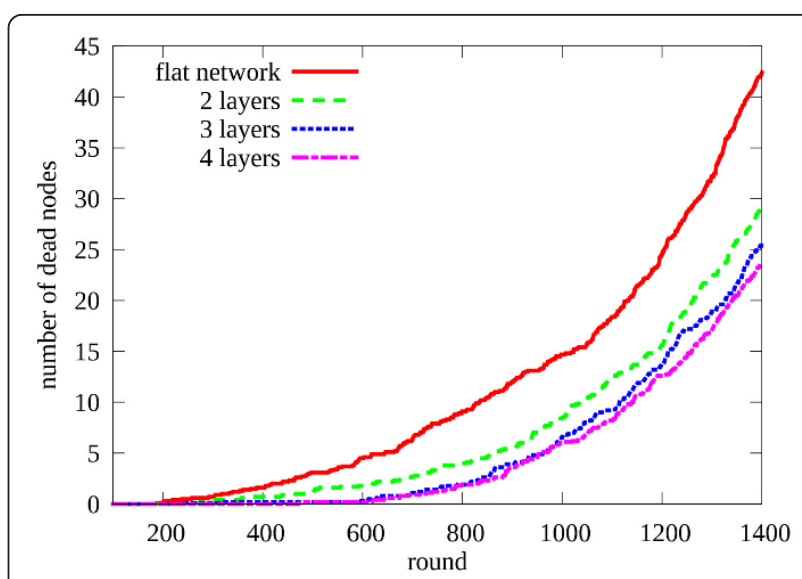

Figure 11 Cumulative number of dead nodes. The change in the number of dead nodes with the passage of time.

from three layers to four. This suggests that the maximum number of layers should be determined by simulation before deployment, since it varies with the number of nodes, their density and distribution.

\section{Conclusions}

We have proposed a new TC scheme for long-term WSNs. In this scheme, each node deter-mines its own layer, which depends on its residual energy and the current network status. This allows the WSN to balance itself by allocating energy-intensive roles to energy-rich nodes, thus extending the network lifetime.

As sensors become more efficient, long-term WSNs will become more common, and require more sophisticated TC, which we believe can be built on the adaptive layer-based scheme reported in this article.

\section{Acknowledgements}

This research was supported partly by Basic Science Research Program through the National Research Foundation of Korea(NRF) funded by the Ministry of Education, Science and Technology(2011-0012996), partly by the Technology Innovation Program funded by the Ministry of Knowledge Economy (MKE) of Korea(10039239), and in part by the ICT at Seoul National University.

\section{Author details}

${ }^{1}$ School of Computer Science and Engineering, Seoul National University, Seoul, Korea ${ }^{2}$ School of Electronic Engineering, Soongsil University, Seoul, Korea

\section{Competing interests}

The authors declare that they have no competing interests.

Received: 30 October 2011 Accepted: 9 May 2012

Published: 9 May 2012

\section{References}

1. Y Tseng, SY Ni, YS Chen, JP Sheu, The broadcast storm problem in a mobile ad hoc network. Wireless networks. 8(2), 153-167 (2002). doi:10.1023/ A:1013763825347

2. B Chen, K Jamieson, H Balakrishnan, R Morris, Span: an energy-efficient coordination algorithm for topology maintenance in ad hoc wireless 
networks. Wireless Networks. 8(5), 481-494 (2002). doi:10.1023/ A:1016542229220

3. $Y X u, J$ Heidemann, D Estrin, Geography-informed energy conservation for ad hoc routing. in Proceedings of the 7th Annual International Conference on Mobile Computing and Networking: Rome, ACM 2001 70-84 (16-21 July 2001)

4. O Younis, S Fahmy, HEED: a hybrid, energy-efficient, distributed clustering approach for ad hoc sensor networks. Mobile Computing, IEEE Transactions on. 3(4), 366-379 (2004). doi:10.1109/TMC.2004.41

5. S Lindsey, C Raghavendra, PEGASIS: Power-efficient gathering in sensor information systems. in Proceedings of Aerospace Conference Proceedings Montana, IEEE 2002. 3, 1125-1130 (9-16 March 2002)

6. J Li, P Mohapatra, An analytical model for the energy hole problem in many-to-one sensor networks. in Proceedings of IEEE Vehicular Technology Conference: Dallas, IEEE 2005. 62, 2721-2725 (25-28 September 2005)

7. W Heinzelman, A Chandrakasan, H Balakrishnan, Energy-efficient communication protocol for wireless microsensor networks. in Proceedings of the 33rd Annual Hawaii International Conference on System Sciences: Hawaii, IEEE 2000. 2, 10 (4-7 January 2000)

8. E Oyman, C Ersoy, Multiple sink network design problem in large scale wireless sensor networks. in Proceedings of IEEE International Conference on Communications: Paris, IEEE. 6, 3663-3667 (24-June 2004)

9. V Shah-Mansouri, A Hamed Mohsenian Rad, V Wong, Multicommodity lifetime routing for wireless sensor networks with multiple sinks. in Proceedings of IC 2008, IEEE International Conference on Communications: Beijing, IEEE 2008 3225-3229 (19-23 May 2008)

10. A Das, D Dutta, Data acquisition in multiple-sink sensor networks. ACM SIGMOBILE Mobile Computing and Communications Review. 9(3), 82-85 (2005). doi:10.1145/1094549.1094561

11. C Buratti, J Orriss, R Verdone, On the design of tree-based topologies for multi-sink wireless sensor networks. in Proceedings of the NEWCOM-ACORN Workshop: Vienna 20066 (20-22 September 2006)

12. C Buratti, R Verdone, Tree-based topology design for multi-sink wireless sensor networks. in Proceedings of PIMRC 2007, IEEE International Symposium on Personal, Indoor and Mobile Radio Communications: Athens, IEEE 2007 1-5 (2-7 September 2007)

13. C Buratti, F Cuomo, S Luna, U Monaco, J Orriss, R Verdone, Optimum treebased topologies for multi-sink wireless sensor networks using ieee 802.15 . 4. in Proceedings of VTC 2007, IEEE 65th Vehicular Technology Conference: Dublin, IEEE 2007 130-134 (22-25 April 2007)

14. J Kim, S Lee, Spanning tree based topology configuration for multiple-sink wire-less sensor networks. in Proceedings of ICUFN 2009, the first International Conference on Ubiquitous and Future Networks: Hong Kong, IEEE 2009 122-125 (7-9 June 2009)

15. Y Fan, Q Chen, J Yu, Topology control algorithm based on bottleneck node for large-scale WSNs. in Proceedings of CIS 2009, International Conference on Computational Intelligence and Security: Beijing, IEEE 2009. 1, 592-597 (11-14 December 2009)

16. P Ciciriello, L Mottola, G Picco, Efficient routing from multiple sources to multiple sinks in wireless sensor networks. in Proceedings of the 4th European conference on Wireless sensor networks: Delft, Springer-Verlag 2007 34-50 (29-31 January 2007)

17. C Intanagonwiwat, D Estrin, R Govindan, J Heidemann, Impact of network density on data aggregation in wireless sensor networks. in Proceedings of the 22nd International Conference on Distributed Computing Systems: Vienna, IEEE 2002 457-458 (2-5 July 2002)

18. S Sharma, M Rani, Three-layer architecture model (TLAM) for energy conservation in wireless sensor networks. in Proceedings of ICUMT 2009, International Conference on Ultra Modern Telecommunications \& Workshops: St Petersburg, IEEE 2009 1-5 (12-14 October 2009)

19. Z Duan, F Guo, M Deng, M Yu, Shortest Path Routing Protocol for Multilayer Mobile Wireless Sensor Networks. in Proceedings of NSWCTC 2009, International Conference on Networks Security, Wireless Communications and Trusted Computing: Wuhan, IEEE 2009. 2, 106-110 (25-26 April 2009)

20. Z Ming, X Bugong, Layer-based self-organizing topology control for sensor networks. in Proceedings of CCC 2008, 27th Chinese Control Conference: Kunming, IEEE 2008 516-520 (16-18 July 2008)

21. T Melodia, D Pompili, I Akyildiz, Optimal local topology knowledge for energy efficient geographical routing in sensor networks. in Proceedings of INFOCOM 2004, Twenty-third Annual Joint Conference of the IEEE Computer and Communications Societies: Hong Kong, IEEE 2004. 3, 1705-1716 (7-11 March 2004)

22. C Perkins, E Royer, Ad-hoc on-demand distance vector routing. in Proceedings of WM-CSA 1999, IEEE Workshop on Mobile Computing Systems and Applications: New Orleans, IEEE 1999 90-100 (25-26 February 1999)

doi:10.1186/1687-1499-2012-164

Cite this article as: Yoon et al:: Multi-layer topology control for longterm wireless sensor networks. EURASIP Journal on Wireless

Communications and Networking 2012 2012:164.

\section{Submit your manuscript to a SpringerOpen ${ }^{\mathcal{O}}$ journal and benefit from:}

- Convenient online submission

- Rigorous peer review

- Immediate publication on acceptance

- Open access: articles freely available online

- High visibility within the field

- Retaining the copyright to your article

Submit your next manuscript at $\gg$ springeropen.com 